\title{
Association between intraoperative administration of dexamethasone and survival after curative resection for non-small cell lung cancer (NSCLC): A propensity score matching analysis
}

\section{Fang Yan}

Department of Anesthesiology, Sun Yat-sen University Cancer Center

\section{Gang Chen}

Department of Medical Oncology, Sun Yat-sen University Cancer Center Jingdun Xie

Department of Anesthesiology, Sun Yat-sen University Cancer Center Huaqiang Zhou

Department of Medical Oncology, Sun Yat-sen University Cancer Center

\section{Wei Xing}

Department of Anesthesiology, Sun Yat-sen University Cancer Center

\section{Qiang Li}

Department of Anesthesiology, Sun Yat-sen University Cancer Center

\section{Weian Zeng}

Department of Anesthesiology, Sun Yat-sen University Cancer Center https://orcid.org/0000-00026783-2245

\section{Dongtai Chen ( $\nabla$ chendt@sysucc.org.cn )}

\section{Research article}

Keywords: Non-small cell lung cancer, Intraoperative, Dexamethasone, Survival

Posted Date: February 12th, 2020

DOI: https://doi.org/10.21203/rs.2.23364/v1

License: (1) This work is licensed under a Creative Commons Attribution 4.0 International License. Read Full License 


\section{Abstract}

Background: Few studies have suggested the correlation between intraoperative dexamethasone and oncological outcomes in non-small cell lung cancer (NSCLC) patients with radical resection. The existing data are inconsistent and inadequate, and more evidence is needed. We therefore undertook a propensitymatched cohort study to investigate the correlation. Methods: 832 patients with stage I to Illa NSCLC who went through a curative resection between January 2008 and December 2013 were enrolled in our study. Propensity-score matching analysis created a population of 206 patients in the non-DEX group and 103 patients in the DEX group. Cox regression analyses were applied to compare the disease-free survival (DFS) and overall survival (OS) between patients who did not and did receive dexamethasone in the propensity score-matched cohort, as well as in the certain patients with high-risk factors of postoperative nausea and vomiting. Results: After propensity score matching, intraoperative dexamethasone was not significantly associated with DFS (HR: 1.014, 95\%Cl: 0.786-1.309, P = 0.913) and OS (HR: 1.221, 95\%Cl: $0.905-1.647, \mathrm{P}=0.191)$. Multivariable cox regression analysis revealed that intraoperative dexamethasone was not still associated with DFS and OS after curative resection for NSCLC. In the subgroup analysis, intraoperative dexamethasone was significantly associated with improved DFS (HR: $0.20,95 \% \mathrm{Cl}: 0.04-0.92, \mathrm{P}=0.038$ ) in the group of anesthetic time less than 2 hours. In the subgroup of VATS, intraoperative dexamethasone was significantly associated with prolonged OS (HR: $0.53,95 \% \mathrm{Cl}$ : $0.30-0.92, P=0.023)$. Conclusion: There was no correlation between intraoperative administration of dexamethasone and survival in NSCLC patients after curative surgery. While patients given intraoperative dexamethasone had better disease-free survival compared with patients not given intraoperative dexamethasone in the subgroup of anesthetic time less than 2 hours. Intraoperative administration of dexamethasone may improve overall survival in the subgroup of VATS. Our results indicate that intraoperative administration of dexamethasone is probably favorable in the aforementioned populations.

\section{Background}

Disease recurrence and metastasis are the fatal hallmark of cancer[1]. Several factors, such as cancer surgery, subsequent therapy and host immune function, affect risk of recurrence and metastasis[2-4]. Major operations may facilitate tumor to release more tumor cells into circulatory system and increase the chance of metastasis[5], but host immune defense system plays an important role in killing tumor cells[6]. Experimental results show that perioperatively damaged immune function augments the possibility of cancer recurrence[7]. Therefore, perioperative period may be crucial.

Many perioperative managements, such as blood transfusion, epidural analgesia and decreased opioid intake, affect cancer outcomes[8-10]. Dexamethasone can decrease the risk of postoperative nausea and vomiting, especially for patients who meet the high-risk factors including female, nonsmoking status, long duration of anesthesia, thoracoscope, laparoscopy, inhaled anesthetics and so on[11, 12].

Dexamethasone as an immunosuppressive drug may have a deleterious effect on cancer recurrence or 
patient overall survival after radical surgery[13]. Depression of natural killer cell function have been supposed to be the mechanism by which dexamethasone can depress host immune defense system[14].

One previous study noted that perioperative dexamethasone may have an advantageous impact on the long-term survival for non-small-cell lung cancer (NSCLC) patients with cancer radical surgery[15]. Another study showed that perioperative dexamethasone was not associated with recurrence-free survival and overall survival after curative surgery for NSCLC[16]. However, these data are inconsistent and inadequate, and more scientific studies are needed. As numerous patients who have lung cancer resection receive intraoperative dexamethasone for prevention of postoperative nausea and vomiting, the effect of dexamethasone on survival is a vital clinical problem.

To address this issue, we undertook a propensity score matching analysis, which was adjusted for strong prognostic factors, to assess the correlation between intraoperatively administration of dexamethasone and survival in NSCLC patients undergoing surgical resection. Specifically, the aim of subgroup analysis was to investigate whether intraoperatively administration of dexamethasone was associated with better or poorer prognosis in the certain patients with high-risk factors of postoperative nausea and vomiting.

\section{Methods}

The study was approved by the research data declaration (RDD) of Sun Yat-sen University cancer Center.

\section{Study participants}

Clinicopathological characteristics of all patients who had lung cancer resection were collected from electronic medical records at Sun Yat-sen University cancer Center between January 2008 and December 2013. The exclusion criteria were as follows: benign lung tumor or small cell lung cancer, metastatic lung cancer, other malignancy, history of lung surgery, bilateral lung cancer, American Society of Anesthesiologists (ASA) physical status equal to or greater than IV, receiving corticosteroids due to chronic obstructive pulmonary disease (COPD)/asthma exacerbation/ inflammatory bowel disease, TNM

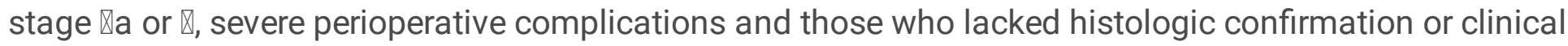
details $[17,18]$. All enrolled patients were divided into two groups on the basis of whether they received dexamethasone during lung cancer resection. Whether patients receive dexamethasone depends on the preference of anesthesiologists. Patients in the dexamethasone group (DEX group) received dexamethasone, and those in the non-dexamethasone group (non-DEX group) didn't receive dexamethasone.

\section{Patient characteristics and Outcome}

The following data were extracted from electronic medical records: age, gender, height, weight, smoking status, co-morbid illness, TNM stage, histology type, tumor size, ASA physical status, type of anesthesia, anesthetic time, operation methods, transfusion, type of postoperative pain control, date of surgery, chemotherapy and radiation therapy. Co-morbid illnesses consist of coronary heart disease, diabetes 
mellitus (DM), hypertension, hepatitis B, hyperthyroidism or hypothyroidism and chronic obstructive pulmonary disease (COPD). Tumor size was the largest diameter of tumor mass.

The primary endpoint of our study was disease-free survival (DFS) and overall survival (OS). DFS was the interval between the date of surgery and the date of relapse of lung cancer, metastasis or death. Relapse of lung cancer was defined as locoregional new occurrences of tumor mass and confirmed by imaging or histopathological examination. Metastasis of lung cancer was defined as the dissemination of lung cancer from lung to another part of the body, and confirmed by imaging or histopathological examination. OS was the interval between the date of surgery and the date of death. We recorded the dates of death from the hospital information system (HIS) of Sun Yat-sen University cancer Center. The final follow-up was December 31, 2018. The follow-up period was at least 5 years. Patients lost to followup during the study period were censored.

\section{Statistical Analysis}

Propensity score matching was conducted to balance baseline characteristics between two groups by reducing the potential confounding factors. Propensity score was the possibility of receiving dexamethasone treatment and calculated by logistic regression analysis. Thereafter, a propensity scorematched cohort of patients receiving vs. not receiving dexamethasone was generated at a ratio of $1: 2$ by using the nearest neighbor method with caliper less than 0.01 . We evaluated the efficiency of matching to balance baseline characteristics by using standard differences $[19,20]$. Categorical variables were presented as the number. Continuous variables were presented as the mean or median. The Student's $t$ test or the Mann-Whitney $U$ test were used for comparisons of continuous variables. The chi-square test or the Fisher's exact test were used to compare categorical variables. In the propensity score-matched cohort, disease-free survival (DFS) and overall survival (OS) were compared between the non-DEX and DEX groups, and calculated using the Kaplan-Meier method. Multivariable cox proportional hazards models were conducted to identify other potential confounding factors associated with intraoperatively administration of dexamethasone. Potential confounding factors consisted of age, tumor size, type of anesthesia, transfusion, year of diagnosis, TNM stage, postoperative radiotherapy and chemotherapy. All variables were entered into multivariable cox proportional hazards regression analysis to compare the hazards ratio between the two groups by using the "enter" method. Multivariable cox proportional hazards regression model was then to assess the association between anesthetic variables and survival after lung cancer resection for NSCLC patients. Associations between intraoperatively administration of dexamethasone and high-risk factors of postoperative nausea and vomiting (PONV) for disease-free survival and overall survival was calculated by cox proportional hazard regression analysis and presented as forest plot[21]. Univariable and multivariable analyses were performed using the IBM SPSS Statistics 23.0 (SPSS Inc, Armonk, NY, USA). Propensity score matching analysis was carried out using R software version 3.5.3 (R Project for Statistical Computing, Austria). A two-tailed $P<0.05$ was considered statistically significant.

\section{Results}


A total of 2480 patients who underwent lung cancer resection at Sun Yat-sen University cancer Center were enrolled during the study period (2008-2013). Of these, 823 patients catered to inclusion criteria and were finally brought into our study (Fig. 1). 823 patients fell into two groups: the non-DEX group ( $\mathrm{N}=$ $675,82 \%)$, and the DEX group ( $N=148,18 \%)$. Among 148 patients with intraoperative dexamethasone therapy, $21(14.2 \%)$ received $5 \mathrm{mg}$ of dexamethasone, $6(4.0 \%)$ received $7.5 \mathrm{mg}$ of dexamethasone and $121(81.8 \%)$ received $10 \mathrm{mg}$ of dexamethasone. All patients in the non-DEX group didn't receive any intraoperative glucocorticoids.

The comparisons of patients' characteristics, tumor-related characteristics, anesthetic variables and surgical variables between non-DEX and DEX groups in the entire cohort are illustrated in Table 1. Smoking status, perioperative blood transfusion, postoperative analgesia and chemotherapy were significantly different between two groups $(P<0.05)$. Variables associated with intraoperatively administration of dexamethasone $(P<0.2)$ as presented in Table 1 were entered into multivariable cox proportional regression analysis. The independent risk factors of intraoperatively administration of dexamethasone, including smoking status $(P<0.001)$, blood transfusion $(P=0.005)$, postoperative analgesia $(P=0.001)$ and chemotherapy $(P=0.037)$, were shown in Table 2 . The variables used for matching were smoking status, blood transfusion, postoperative analgesia and postoperative adjuvant chemotherapy. 
Table 1

Comparison of baseline characteristics between the intraoperative dexamethasone (DEX) and non-DEX groups in the entire cohort.

\begin{tabular}{|c|c|c|c|}
\hline Variable & $\begin{array}{l}\text { Non-DEX group } \\
(\mathrm{N}=675)\end{array}$ & $\begin{array}{l}\text { DEX group } \\
(N=148)\end{array}$ & P value \\
\hline Age, years & 60.21 & 59.31 & 0.58 \\
\hline Sex & & & 0.388 \\
\hline Male & 382 & 78 & \\
\hline Female & 293 & 70 & \\
\hline $\mathrm{BMI}, \mathrm{kg} / \mathrm{m} 2$ & 23.02 & 23.12 & 0.05 \\
\hline Smoking status & & & $\nabla 0.001$ * \\
\hline Never & 363 & 116 & \\
\hline Ever & 312 & 32 & \\
\hline Co-morbid illness & & & 0.191 \\
\hline No & 441 & 105 & \\
\hline Yes & 234 & 43 & \\
\hline TNM stage & & & 0.326 \\
\hline ૧ & 157 & 23 & \\
\hline ૧ & 122 & 33 & \\
\hline ૧ & 374 & 87 & \\
\hline Histology type & & & 0.876 \\
\hline Adenocarcinoma & 530 & 113 & \\
\hline Squamous cell carcinoma & 96 & 24 & \\
\hline Large cell carcinoma & 6 & 2 & \\
\hline Others & 43 & 9 & \\
\hline Tumor size, cm & 3.30 & 3.42 & 0.813 \\
\hline ASA score & & & 0.939 \\
\hline$\leq 2$ & 608 & 133 & \\
\hline$₫ 2$ & 67 & 15 & \\
\hline Duration of anesthesia & 3.64 & 4.15 & 0.39 \\
\hline
\end{tabular}




\begin{tabular}{|llll|}
\hline Variable & $\begin{array}{l}\text { Non-DEX group } \\
(\mathbf{N}=675)\end{array}$ & $\begin{array}{l}\text { DEX group } \\
(\mathbf{N}=148)\end{array}$ & P value \\
\hline Type of anesthesia & & 145 & 0.138 \\
\hline Inhalation & 643 & 3 & \\
\hline Intravenous & 32 & & 0.938 \\
\hline Operative approach & & 102 & \\
\hline Thoracotomy & 463 & 46 & \\
\hline VATS & 212 & & $0.001 *$ \\
\hline Blood transfusion & & 141 & \\
\hline No & 573 & 7 & \\
\hline Yes & 102 & & \\
\hline
\end{tabular}


Table 1

Comparison of baseline characteristics between the intraoperative dexamethasone (DEX) and non-DEX groups in the entire cohort. (Continued)

\begin{tabular}{|c|c|c|c|}
\hline Variable & $\begin{array}{l}\text { Non-DEX group } \\
(N=675)\end{array}$ & $\begin{array}{l}\text { DEX group } \\
(N=148)\end{array}$ & $P$ value \\
\hline \multicolumn{3}{|l|}{ Postoperative analgesia } & $\unrhd 0.001^{*}$ \\
\hline No & 43 & 9 & \\
\hline PCIA & 433 & 128 & \\
\hline PCEA & 152 & 11 & \\
\hline \multicolumn{3}{|l|}{ Radiotherapy } & 0.119 \\
\hline No & 539 & 110 & \\
\hline Yes & 134 & 38 & \\
\hline \multicolumn{3}{|l|}{ Chemotherapy } & $0.012^{*}$ \\
\hline No & 323 & 54 & \\
\hline Yes & 352 & 94 & \\
\hline \multicolumn{3}{|l|}{ Year of diagnosis } & 0.777 \\
\hline $2006-2010$ & 445 & 104 & \\
\hline $2011-2015$ & 230 & 44 & \\
\hline \multicolumn{4}{|c|}{$\begin{array}{l}\text { ASA, American Society of Anesthesiologists; BMI, body mass index; Dex, dexamethasone; PCIA, } \\
\text { patient controlled intravenous analgesia; PCEA, patient controlled epidural analgesia; VATS, video- } \\
\text { assisted thoracic surgery. }\end{array}$} \\
\hline
\end{tabular}


Table 2

Multivariable analysis of independent predictive factors of intraoperative dexamethasone.

\begin{tabular}{|llll|}
\hline Independent predictive factor & Wald & P value & HR (95\% Cl) \\
\hline BMI & 0.110 & 0.740 & $0.936(0.635-1.380)$ \\
\hline Smoking status & 20.561 & $<0.001^{\star}$ & $2.716(1.764-4.184)$ \\
\hline Co-morbid illness & 0.999 & 0.318 & $1.232(0.818-1.854)$ \\
\hline Type of anesthesia & 2.411 & 0.120 & $2.653(0.774-9.090)$ \\
\hline Blood transfusion & 8.032 & $0.005^{\star}$ & $3.194(1.431-7.130)$ \\
\hline Postoperative analgesia & 11.386 & $0.001^{\star}$ & $1.973(1.330-2.928)$ \\
\hline Radiotherapy & 2.171 & 0.141 & $0.716(0.459-1.117)$ \\
\hline Chemotherapy & 4.345 & $0.037^{\star}$ & $0.661(0.448-0.976)$ \\
\hline
\end{tabular}

A comparison of baseline characteristics after propensity score matching analysis between the non-DEX group and the DEX group is illustrated in Table 3. Propensity score matching analysis generated a cohort of 206 patients in the non-DEX group and 103 patients in the DEX group. All baseline characteristics between two groups were balanced $(P>0.1)$. The median follow-up period was 44 (interquartile range, 25.5 to 62 ) months for all the matched patients. In the non-DEX group and the DEX group, the median follow-up period was 46 (interquartile range, 25 to 64) months and 42 (interquartile range, 26 to 59) months, respectively. There was no significant difference in the follow-up period among two groups $(\mathrm{P}=$ 0.401). The Kaplan-Meier survival curves demonstrated 1-yr, 3-yr and 5-yr disease-free survival rates in the non-DEX and DEX groups were $70.9 \%, 31.3 \%, 19.6 \%$ and $72.8 \%, 30.1 \%, 15.1 \%$, respectively; While 1-yr, 3-yr and 5-yr overall survival rates in the non-DEX and DEX groups were $91.2 \%, 65.4 \%, 48.1 \%$ and $92.2 \%$, $63.5 \%, 34.5 \%$, respectively. There was no significant difference between the non-DEX group and the DEX group in disease-free survival $(P=0.913)$ or overall survival $(P=0.191)$ (Fig. 2). Multivariable cox proportional hazards regression model for disease-free survival was carried out and revealed no statistically significant association between intraoperative dexamethasone and disease-free survival (HR: 1.057, 95\% Cl: $0.809-1.381, \mathrm{P}=0.686$ ) (Table 4). Then, multivariable cox proportional regression analysis for overall survival was performed and revealed that intraoperative dexamethasone was not associated with poorer overall survival (HR: $0.205,95 \% \mathrm{Cl}: 0.893-1.690, \mathrm{P}=0.191$ ) (Table 5). We also conducted cox proportional regression analyses to identify risk factors for disease-free survival and overall survival. Age over 60 years, advanced TNM stage and usage of chemotherapy were significantly associated with poorer disease-free survival; While age over 60 years, advanced TNM stage and tumor size more than $4 \mathrm{~cm}$ were associated with poorer overall survival. 
Table 3

Comparisons of baseline characteristics between the intraoperative dexamethasone (DEX) and non-DEX groups in the propensity scorematched sample.

\begin{tabular}{|c|c|c|c|}
\hline Variable & $\begin{array}{l}\text { Non-DEX group } \\
(\mathrm{N}=206)\end{array}$ & $\begin{array}{l}\text { DEX group } \\
(N=103)\end{array}$ & $P$ value \\
\hline Age, years & 60.2 & 59.3 & 0.889 \\
\hline Sex & & & 0.374 \\
\hline Male & 117 & 53 & \\
\hline Female & 89 & 50 & \\
\hline $\mathrm{BMl}, \mathrm{kg} / \mathrm{m} 2$ & 23.10 & 23.26 & 0.32 \\
\hline Smoking status & & & 0.84 \\
\hline Never & 166 & 82 & \\
\hline Ever & 40 & 21 & \\
\hline Co-morbid illness & & & 0.129 \\
\hline No & 128 & 73 & \\
\hline Yes & 78 & 30 & \\
\hline TNM stage & & & 0.459 \\
\hline 口 & 32 & 11 & \\
\hline 口 & 46 & 22 & \\
\hline 口 & 128 & 70 & \\
\hline Histology type & & & 0.669 \\
\hline Adenocarcinoma & 169 & 79 & \\
\hline Squamous cell carcinoma & 27 & 19 & \\
\hline Large cell carcinoma & 2 & 1 & \\
\hline Others & 8 & 4 & \\
\hline Tumor size, cm & 3.49 & 3.48 & 0.626 \\
\hline ASA score & & & 0.415 \\
\hline$\leq 2$ & 188 & 91 & \\
\hline 『2 & 18 & 12 & \\
\hline
\end{tabular}




\begin{tabular}{|c|c|c|c|}
\hline Variable & $\begin{array}{l}\text { Non-DEX group } \\
(\mathrm{N}=206)\end{array}$ & $\begin{array}{l}\text { DEX group } \\
(N=103)\end{array}$ & $P$ value \\
\hline Duration of anesthesia & 3.77 & 3.98 & 0.459 \\
\hline Type of anesthesia & & & 0.122 \\
\hline Inhalation & 191 & 100 & \\
\hline Intravenous & 15 & 3 & \\
\hline Operative approach & & & 1.0 \\
\hline Thoracotomy & 138 & 69 & \\
\hline VATS & 68 & 34 & \\
\hline Blood transfusion & & & 0.311 \\
\hline No & 202 & 99 & \\
\hline Yes & 4 & 4 & \\
\hline Postoperative analgesia & & & 0.626 \\
\hline No & 15 & 7 & \\
\hline PCIA & 168 & 88 & \\
\hline PCEA & 23 & 8 & \\
\hline
\end{tabular}


Table 3

Comparisons of baseline characteristics between the intraoperative dexamethasone (DEX) and non-DEX groups in the propensity score-matched sample. (Continued)

\begin{tabular}{|llll|}
\hline Variable & $\begin{array}{l}\text { Non-DEX group } \\
(\mathbf{N}=\mathbf{2 0 6})\end{array}$ & $\begin{array}{l}\text { DEX group } \\
(\mathbf{N}=103)\end{array}$ & P value \\
\hline No & 150 & 71 & \\
\hline Yes & 56 & 32 & 0.397 \\
\hline Chemotherapy & & 32 & \\
\hline No & 74 & 71 & \\
\hline Yes & 132 & 73 & \\
\hline Year of diagnosis & 128 & 30 & \\
\hline $2006-2010$ & 78 & & \\
\hline 2011-2015 & & & \\
\hline $\begin{array}{l}\text { ASA, American Society of Anesthesiologists; BMl, body mass index; Dex, dexamethasone; PCIA, } \\
\text { patient controlled intravenous analgesia; PCEA, patient controlled epidural analgesia; VATS, video- } \\
\text { assisted thoracic surgery. }\end{array}$ & & \\
\hline P-value less than 0.05 was considered statistically significant. & \\
\hline
\end{tabular}


Table 4

Univariable and multivariable Cox regression analyses for disease-free survival in the propensity matched cohort.

\begin{tabular}{|c|c|c|c|c|c|c|}
\hline \multirow[t]{2}{*}{ Variable } & \multicolumn{3}{|c|}{ Univariable } & \multicolumn{3}{|c|}{ Multivariable } \\
\hline & $\mathrm{HR}$ & $95 \% \mathrm{Cl}$ & $\begin{array}{l}P \\
\text { value }\end{array}$ & $H R$ & $95 \% \mathrm{Cl}$ & $\begin{array}{l}P \\
\text { value }\end{array}$ \\
\hline \multicolumn{7}{|c|}{ Dexamethasone } \\
\hline No & \multicolumn{3}{|c|}{ Reference } & \multicolumn{3}{|c|}{ Reference } \\
\hline Yes & 1.014 & $\begin{array}{l}0.786- \\
1.309\end{array}$ & 0.913 & 1.065 & $\begin{array}{l}0.814- \\
1.391\end{array}$ & 0.647 \\
\hline \multicolumn{7}{|c|}{ Age, years } \\
\hline Age $\leq 60$ & \multicolumn{3}{|c|}{ Reference } & \multicolumn{3}{|c|}{ Reference } \\
\hline Age $₫ 60$ & 1.206 & $\begin{array}{l}0.947- \\
1.535\end{array}$ & 0.129 & 1.362 & $\begin{array}{l}1.049- \\
1.770\end{array}$ & 0.021 \\
\hline \multicolumn{7}{|l|}{ Sex } \\
\hline Female & \multicolumn{3}{|c|}{ Reference } & \multicolumn{3}{|c|}{ Reference } \\
\hline Male & 0.859 & $\begin{array}{l}0.674- \\
1.095\end{array}$ & 0.219 & 0.886 & $\begin{array}{l}0.678- \\
1.157\end{array}$ & 0.374 \\
\hline \multicolumn{7}{|c|}{$\mathrm{BMI}, \mathrm{kg} / \mathrm{m}^{2}$} \\
\hline$\leq 24$ & \multicolumn{3}{|c|}{ Reference } & \multicolumn{3}{|c|}{ Reference } \\
\hline$\varangle 24$ & 0.988 & $\begin{array}{l}0.771- \\
1.268\end{array}$ & 0.926 & 0.998 & $\begin{array}{l}0.767- \\
1.298\end{array}$ & 0.988 \\
\hline \multicolumn{7}{|c|}{ Smoking status } \\
\hline Never & \multicolumn{3}{|c|}{ Reference } & \multicolumn{3}{|c|}{ Reference } \\
\hline Ever & 0.971 & $\begin{array}{l}0.720- \\
1.310\end{array}$ & 0.847 & 0.898 & $\begin{array}{l}0.648- \\
1.245\end{array}$ & 0.519 \\
\hline \multicolumn{7}{|c|}{ Co-morbid illness } \\
\hline No & \multicolumn{3}{|c|}{ Reference } & \multicolumn{3}{|c|}{ Reference } \\
\hline Yes & 0.948 & $\begin{array}{l}0.742- \\
1.211\end{array}$ & 0.668 & 0.900 & $\begin{array}{l}0.688- \\
1.178\end{array}$ & 0.444 \\
\hline \multicolumn{7}{|c|}{ TNM stage } \\
\hline प & Refere & & & Refere & & \\
\hline
\end{tabular}




\begin{tabular}{|c|c|c|c|c|c|c|}
\hline \multirow{2}{*}{ Variable } & \multicolumn{3}{|c|}{ Univariable } & \multicolumn{3}{|c|}{ Multivariable } \\
\hline & 0.485 & $\begin{array}{l}0.331- \\
0.710\end{array}$ & $\nabla 0.001$ & 0.510 & $\begin{array}{l}0.334- \\
0.778\end{array}$ & 0.008 \\
\hline प & 0.741 & $\begin{array}{l}0.548- \\
1.000\end{array}$ & 0.051 & 0.879 & $\begin{array}{l}0.636- \\
1.217\end{array}$ & 0.002 \\
\hline \multicolumn{7}{|l|}{ Histology type } \\
\hline Adenocarcinoma & \multicolumn{3}{|c|}{ Reference } & \multicolumn{3}{|c|}{ Reference } \\
\hline $\begin{array}{l}\text { Squamous cell } \\
\text { carcinoma }\end{array}$ & 0.898 & $\begin{array}{l}0.490- \\
1.646\end{array}$ & 0.727 & 0.816 & $\begin{array}{l}0.430- \\
1.550\end{array}$ & 0.535 \\
\hline Large cell carcinoma & 0.640 & $\begin{array}{l}0.327- \\
1.253\end{array}$ & 0.193 & 0.614 & $\begin{array}{l}0.309- \\
1.219\end{array}$ & 0.163 \\
\hline Others & 1.646 & $\begin{array}{l}0.459- \\
5.906\end{array}$ & 0.445 & 1.536 & $\begin{array}{l}0.392- \\
6.014\end{array}$ & 0.538 \\
\hline \multicolumn{7}{|l|}{ Tumor size, cm } \\
\hline$\leq 4$ & \multicolumn{3}{|c|}{ Reference } & \multicolumn{3}{|c|}{ Reference } \\
\hline$\otimes 4$ & 1.017 & $\begin{array}{l}0.768- \\
1.346\end{array}$ & 0.907 & 1.006 & $\begin{array}{l}0.732- \\
1.382\end{array}$ & 0.972 \\
\hline \multicolumn{7}{|l|}{ ASA score } \\
\hline$\leq 2$ & \multicolumn{3}{|c|}{ Reference } & \multicolumn{3}{|c|}{ Reference } \\
\hline$\otimes 2$ & 1.002 & $\begin{array}{l}0.781- \\
1.285\end{array}$ & 0.986 & 1.023 & $\begin{array}{l}0.783- \\
1.337\end{array}$ & 0.868 \\
\hline
\end{tabular}


Table 4

Univariable and multivariable Cox regression analyses for disease-free survival in the propensity matched cohort. (Continued)

\begin{tabular}{|c|c|c|c|c|c|c|}
\hline \multirow[t]{2}{*}{ Variable } & \multicolumn{3}{|c|}{ Univariable } & \multicolumn{3}{|c|}{ Multivariable } \\
\hline & $\mathrm{HR}$ & $95 \% \mathrm{Cl}$ & $\begin{array}{l}P \\
\text { value }\end{array}$ & $\mathrm{HR}$ & $95 \% \mathrm{Cl}$ & $\begin{array}{l}P \\
\text { value }\end{array}$ \\
\hline \multicolumn{7}{|c|}{ Anesthetic time, $\mathrm{hr}$} \\
\hline$\leq 2$ & \multicolumn{3}{|c|}{ Reference } & \multicolumn{3}{|c|}{ Reference } \\
\hline$\bigotimes 2$ & 0.667 & $\begin{array}{l}0.407- \\
1.092\end{array}$ & 0.107 & 0.737 & $\begin{array}{l}0.432- \\
1.258\end{array}$ & 0.263 \\
\hline \multicolumn{7}{|c|}{ Type of anesthesia } \\
\hline Inhalation & \multicolumn{3}{|c|}{ Reference } & \multicolumn{3}{|c|}{ Reference } \\
\hline Intravenous & 0.861 & $\begin{array}{l}0.493- \\
1.505\end{array}$ & 0.600 & 0.987 & $\begin{array}{l}0.535- \\
1.818\end{array}$ & 0.966 \\
\hline \multicolumn{7}{|c|}{ Operative approach } \\
\hline Thoracotomy & \multicolumn{3}{|c|}{ Reference } & \multicolumn{3}{|c|}{ Reference } \\
\hline VATS & 0.802 & $\begin{array}{l}0.617- \\
1.041\end{array}$ & 0.097 & 0.813 & $\begin{array}{l}0.611- \\
1.082\end{array}$ & 0.156 \\
\hline \multicolumn{7}{|c|}{ Blood transfusion } \\
\hline No & \multicolumn{3}{|c|}{ Reference } & \multicolumn{3}{|c|}{ Reference } \\
\hline Yes & 1.306 & $\begin{array}{l}0.645- \\
2.643\end{array}$ & 0.459 & 0.990 & $\begin{array}{l}0.471- \\
2.081\end{array}$ & 0.979 \\
\hline \multicolumn{7}{|l|}{$\begin{array}{l}\text { Postoperative } \\
\text { analgesia }\end{array}$} \\
\hline No & \multicolumn{3}{|c|}{ Reference } & \multicolumn{3}{|c|}{ Reference } \\
\hline PCIA & 1.200 & $\begin{array}{l}0.657- \\
2.192\end{array}$ & 0.554 & 1.236 & $\begin{array}{l}0.668- \\
2.288\end{array}$ & 0.500 \\
\hline PCEA & 1.226 & $\begin{array}{l}0.804- \\
1.868\end{array}$ & 0.344 & 1.160 & $\begin{array}{l}0.751- \\
1.791\end{array}$ & 0.503 \\
\hline \multicolumn{7}{|l|}{ Radiotherapy } \\
\hline No & \multicolumn{3}{|c|}{ Reference } & \multicolumn{3}{|c|}{ Reference } \\
\hline \multicolumn{7}{|c|}{$\begin{array}{l}\text { ASA, American Society of Anesthesiologists; } \mathrm{BMI} \text {, body mass index; } \mathrm{Cl} \text {, confidence index; Dex, } \\
\text { dexamethasone; HR, hazard ratio; PCIA, patient controlled intravenous analgesia; PCEA, patient } \\
\text { controlled epidural analgesia; VATS, video-assisted thoracic surgery. }\end{array}$} \\
\hline
\end{tabular}




\begin{tabular}{|c|c|c|c|c|c|c|}
\hline \multirow{2}{*}{$\begin{array}{l}\text { Variable } \\
\text { Yes }\end{array}$} & \multicolumn{3}{|c|}{ Univariable } & \multicolumn{3}{|c|}{ Multivariable } \\
\hline & 1.418 & $\begin{array}{l}1.090- \\
1.844\end{array}$ & 0.009 & 1.309 & $\begin{array}{l}0.987- \\
1.736\end{array}$ & 0.062 \\
\hline \multicolumn{7}{|c|}{ Chemotherapy } \\
\hline No & \multicolumn{3}{|c|}{ Reference } & \multicolumn{3}{|c|}{ Reference } \\
\hline Yes & 1.489 & $\begin{array}{l}1.153- \\
1.923\end{array}$ & 0.002 & 1.417 & $\begin{array}{l}1.073- \\
1.871\end{array}$ & 0.014 \\
\hline \multicolumn{7}{|c|}{ Year of diagnosis } \\
\hline $2006-2010$ & \multicolumn{3}{|c|}{ Reference } & \multicolumn{3}{|c|}{ Reference } \\
\hline $2011-2015$ & 0.987 & $\begin{array}{l}0.925- \\
1.054\end{array}$ & 0.698 & 0.916 & $\begin{array}{l}0.674- \\
1.243\end{array}$ & 0.573 \\
\hline \multicolumn{7}{|c|}{$\begin{array}{l}\text { ASA, American Society of Anesthesiologists; BMI, body mass index; } \mathrm{Cl} \text {, confidence index; Dex, } \\
\text { dexamethasone; HR, hazard ratio; PCIA, patient controlled intravenous analgesia; PCEA, patient } \\
\text { controlled epidural analgesia; VATS, video-assisted thoracic surgery. }\end{array}$} \\
\hline \multicolumn{7}{|c|}{ P-value less than 0.05 was considered statistically significant. } \\
\hline
\end{tabular}


Table 5

Univariable and multivariable Cox regression analyses for overall survival in the propensity matched cohort.

\begin{tabular}{|c|c|c|c|c|c|c|}
\hline \multirow[t]{2}{*}{ Variable } & \multicolumn{3}{|c|}{ Univariable } & \multicolumn{3}{|c|}{ Multivariable } \\
\hline & $\mathrm{HR}$ & $95 \% \mathrm{Cl}$ & $\begin{array}{l}\mathrm{P} \\
\text { value }\end{array}$ & $\mathrm{HR}$ & $95 \% \mathrm{Cl}$ & $\begin{array}{l}\mathrm{P} \\
\text { value }\end{array}$ \\
\hline \multicolumn{7}{|c|}{ Dexamethasone } \\
\hline No & \multicolumn{3}{|c|}{ Reference } & \multicolumn{3}{|c|}{ Reference } \\
\hline Yes & 1.221 & $\begin{array}{l}0.905- \\
1.647\end{array}$ & 0.191 & 1.219 & $\begin{array}{l}0.887- \\
1.677\end{array}$ & 0.223 \\
\hline \multicolumn{7}{|c|}{ Age, years } \\
\hline Age $\leq 60$ & \multicolumn{3}{|c|}{ Reference } & \multicolumn{3}{|c|}{ Reference } \\
\hline Age $₫ 60$ & 1.437 & $\begin{array}{l}1.076- \\
1.920\end{array}$ & 0.014 & 1.423 & $\begin{array}{l}1.035- \\
1.956\end{array}$ & 0.030 \\
\hline \multicolumn{7}{|l|}{ Sex } \\
\hline Female & \multicolumn{3}{|c|}{ Reference } & \multicolumn{3}{|c|}{ Reference } \\
\hline Male & 1.057 & $\begin{array}{l}0.791- \\
1.414\end{array}$ & 0.706 & 1.017 & $\begin{array}{l}0.739- \\
1.400\end{array}$ & 0.918 \\
\hline \multicolumn{7}{|c|}{$\mathrm{BMI}, \mathrm{kg} / \mathrm{m}^{2}$} \\
\hline$\leq 24$ & \multicolumn{3}{|c|}{ Reference } & \multicolumn{3}{|c|}{ Reference } \\
\hline$\varangle 24$ & 0.997 & $\begin{array}{l}0.740- \\
1.343\end{array}$ & 0.983 & 1.013 & $\begin{array}{l}0.732- \\
1.402\end{array}$ & 0.938 \\
\hline \multicolumn{7}{|c|}{ Smoking status } \\
\hline Never & \multicolumn{3}{|c|}{ Reference } & \multicolumn{3}{|c|}{ Reference } \\
\hline Ever & 1.367 & $\begin{array}{l}0.975- \\
1.919\end{array}$ & 0.07 & 1.278 & $\begin{array}{l}0.881- \\
1.855\end{array}$ & 0.196 \\
\hline \multicolumn{7}{|c|}{ Co-morbid illness } \\
\hline No & \multicolumn{3}{|c|}{ Reference } & \multicolumn{3}{|c|}{ Reference } \\
\hline Yes & 0.878 & $\begin{array}{l}0.651- \\
1.183\end{array}$ & 0.392 & 0.846 & $\begin{array}{l}0.611- \\
1.171\end{array}$ & 0.313 \\
\hline \multicolumn{7}{|c|}{ TNM stage } \\
\hline ( & Refere & & & Refere & & \\
\hline
\end{tabular}




\begin{tabular}{|c|c|c|c|c|c|c|}
\hline \multirow{2}{*}{ Variable } & \multicolumn{3}{|c|}{ Univariable } & \multicolumn{3}{|c|}{ Multivariable } \\
\hline & 0.754 & $\begin{array}{l}0.489- \\
1.162\end{array}$ & 0.200 & 0.560 & $\begin{array}{l}0.347- \\
0.904\end{array}$ & 0.033 \\
\hline प & 0.753 & $\begin{array}{l}0.521- \\
1.088\end{array}$ & 0.130 & 0.715 & $\begin{array}{l}0.482- \\
1.059\end{array}$ & 0.018 \\
\hline \multicolumn{7}{|l|}{ Histology type } \\
\hline Adenocarcinoma & \multicolumn{3}{|c|}{ Reference } & \multicolumn{3}{|c|}{ Reference } \\
\hline $\begin{array}{l}\text { Squamous cell } \\
\text { carcinoma }\end{array}$ & 0.609 & $\begin{array}{l}0.310- \\
1.196\end{array}$ & 0.150 & 0.763 & $\begin{array}{l}0.369- \\
1.578\end{array}$ & 0.466 \\
\hline Large cell carcinoma & 0.741 & $\begin{array}{l}0.353- \\
1.553\end{array}$ & 0.427 & 0.693 & $\begin{array}{l}0.323- \\
1.487\end{array}$ & 0.347 \\
\hline Others & 2.359 & $\begin{array}{l}0.635- \\
8.759\end{array}$ & 0.200 & 1.799 & $\begin{array}{l}0.425- \\
7.624\end{array}$ & 0.425 \\
\hline \multicolumn{7}{|l|}{ Tumor size, cm } \\
\hline$\leq 4$ & \multicolumn{3}{|c|}{ Reference } & \multicolumn{3}{|c|}{ Reference } \\
\hline$\otimes 4$ & 1.784 & $\begin{array}{l}1.303- \\
2.444\end{array}$ & $\otimes 0.001$ & 1.630 & $\begin{array}{l}1.131- \\
2.349\end{array}$ & 0.009 \\
\hline \multicolumn{7}{|l|}{ ASA score } \\
\hline$\leq 2$ & \multicolumn{3}{|c|}{ Reference } & \multicolumn{3}{|c|}{ Reference } \\
\hline$\otimes 2$ & 1.263 & $\begin{array}{l}0.943- \\
1.693\end{array}$ & 0.118 & 1.125 & $\begin{array}{l}0.824- \\
1.534\end{array}$ & 0.459 \\
\hline
\end{tabular}


Table 5

Univariable and multivariable Cox regression analyses for overall survival in the propensity matched cohort. (Continued)

\begin{tabular}{|c|c|c|c|c|c|c|}
\hline \multirow[t]{2}{*}{ Variable } & \multicolumn{3}{|c|}{ Univariable } & \multicolumn{3}{|c|}{ Multivariable } \\
\hline & $\mathrm{HR}$ & $95 \% \mathrm{Cl}$ & $\begin{array}{l}\mathrm{P} \\
\text { value }\end{array}$ & $\mathrm{HR}$ & $95 \% \mathrm{Cl}$ & $\begin{array}{l}\mathrm{P} \\
\text { value }\end{array}$ \\
\hline \multicolumn{7}{|c|}{ Anesthetic time, hr } \\
\hline$\leq 2$ & \multicolumn{3}{|c|}{ Reference } & \multicolumn{3}{|c|}{ Reference } \\
\hline$₫ 2$ & 0.735 & $\begin{array}{l}0.409- \\
1.321\end{array}$ & 0.303 & 0.590 & $\begin{array}{l}0.312- \\
1.114\end{array}$ & 0.104 \\
\hline \multicolumn{7}{|c|}{ Type of anesthesia } \\
\hline Inhalation & \multicolumn{3}{|c|}{ Reference } & \multicolumn{3}{|c|}{ Reference } \\
\hline Intravenous & 0.344 & $\begin{array}{l}0.128- \\
0.928\end{array}$ & 0.035 & 0.470 & $\begin{array}{l}0.165- \\
1.332\end{array}$ & 0.155 \\
\hline \multicolumn{7}{|c|}{ Operative approach } \\
\hline Thoracotomy & \multicolumn{3}{|c|}{ Reference } & \multicolumn{3}{|c|}{ Reference } \\
\hline VATS & 0.755 & $\begin{array}{l}0.547- \\
1.041\end{array}$ & 0.086 & 0.935 & $\begin{array}{l}0.657- \\
1.332\end{array}$ & 0.711 \\
\hline \multicolumn{7}{|c|}{ Blood transfusion } \\
\hline No & \multicolumn{3}{|c|}{ Reference } & \multicolumn{3}{|c|}{ Reference } \\
\hline Yes & 2.257 & $\begin{array}{l}1.057- \\
4.815\end{array}$ & 0.035 & 2.151 & $\begin{array}{l}0.947- \\
4.885\end{array}$ & 0.067 \\
\hline \multicolumn{7}{|l|}{$\begin{array}{l}\text { Postoperative } \\
\text { analgesia }\end{array}$} \\
\hline No & \multicolumn{3}{|c|}{ Reference } & \multicolumn{3}{|c|}{ Reference } \\
\hline PCIA & 1.034 & $\begin{array}{l}0.489- \\
2.187\end{array}$ & 0.931 & 0.984 & $\begin{array}{l}0.457- \\
2.121\end{array}$ & 0.968 \\
\hline PCEA & 1.189 & $\begin{array}{l}0.711- \\
1.989\end{array}$ & 0.510 & 1.065 & $\begin{array}{l}0.626- \\
1.814\end{array}$ & 0.816 \\
\hline \multicolumn{7}{|l|}{ Radiotherapy } \\
\hline No & \multicolumn{3}{|c|}{ Reference } & \multicolumn{3}{|c|}{ Reference } \\
\hline \multicolumn{7}{|c|}{$\begin{array}{l}\text { ASA, American Society of Anesthesiologists; BMI, body mass index; } \mathrm{Cl} \text {, confidence index; Dex, } \\
\text { dexamethasone; HR, hazard ratio; PCIA, patient controlled intravenous analgesia; PCEA, patient } \\
\text { controlled epidural analgesia; VATS, video-assisted thoracic surgery. }\end{array}$} \\
\hline
\end{tabular}




\begin{tabular}{|c|c|c|c|c|c|c|}
\hline \multirow{2}{*}{$\begin{array}{l}\text { Variable } \\
\text { Yes }\end{array}$} & \multicolumn{3}{|c|}{ Univariable } & \multicolumn{3}{|c|}{ Multivariable } \\
\hline & 0.944 & $\begin{array}{l}0.686- \\
1.298\end{array}$ & 0.723 & 0.821 & $\begin{array}{l}0.581- \\
1.160\end{array}$ & 0.264 \\
\hline \multicolumn{7}{|c|}{ Chemotherapy } \\
\hline No & \multicolumn{3}{|c|}{ Reference } & \multicolumn{3}{|c|}{ Reference } \\
\hline Yes & 1.183 & $\begin{array}{l}0.873- \\
1.601\end{array}$ & 0.279 & 1.060 & $\begin{array}{l}0.760- \\
1.479\end{array}$ & 0.730 \\
\hline \multicolumn{7}{|c|}{ Year of diagnosis } \\
\hline $2006-2010$ & \multicolumn{3}{|c|}{ Reference } & \multicolumn{3}{|c|}{ Reference } \\
\hline $2011-2015$ & 0.894 & $\begin{array}{l}0.825- \\
0.968\end{array}$ & 0.006 & 0.693 & $\begin{array}{l}0.471- \\
1.020\end{array}$ & 0.063 \\
\hline \multicolumn{7}{|c|}{$\begin{array}{l}\text { ASA, American Society of Anesthesiologists; BMI, body mass index; } \mathrm{Cl} \text {, confidence index; Dex, } \\
\text { dexamethasone; HR, hazard ratio; PCIA, patient controlled intravenous analgesia; PCEA, patient } \\
\text { controlled epidural analgesia; VATS, video-assisted thoracic surgery. }\end{array}$} \\
\hline \multicolumn{7}{|c|}{ P-value less than 0.05 was considered statistically significant. } \\
\hline
\end{tabular}

These propensity score-matched patients were stratified according to the high-risk factors of postoperative nausea and vomiting. Subgroup analysis was performed to explore whether intraoperative dexamethasone affected survival in some certain subgroup. Figure 3 reported a significant correlation between intraoperatively administration of dexamethasone and improved disease-free survival in the subgroup of anesthetic time less than $2 \mathrm{hrs}$ (HR: $0.20,95 \% \mathrm{Cl}: 0.04-0.92, \mathrm{P}=0.038$ ). Meanwhile, there was a significant association between intraoperatively administration of dexamethasone and longer overall survival in the subgroup of VATS (HR: $0.53,95 \% \mathrm{Cl}: 0.30-0.92, \mathrm{P}=0.023$ ).

\section{Discussion}

Our study found no correlation between intraoperatively administration of dexamethasone and survival in NSCLC patients after lung cancer resection. While patients given dexamethasone had better disease-free survival in the subgroup of anesthetic time less than 2 hours. Intraoperative administration of dexamethasone may improve overall survival in the subgroup of VATS. Our results indicate that intraoperative dexamethasone, commonly used for prevention of postoperative nausea and vomiting (PONV), do not augment cancer recurrence and affect overall survival in non-small cell lung cancer patients having lung cancer resection. However, intraoperative administration of systemic dexamethasone is probably favorable in the aforementioned populations.

Glucocorticoids (GCs), such as dexamethasone, can arrest growth or induce apoptosis in lymphocytes[22]. In leukemia and lymphoma, dexamethasone is the cornerstone of treatment for all lymphatic cancers and hematopoietic malignancies[23]. Moreover, dexamethasone performs a variety of 
functions, including preventing postoperative nausea and vomiting, reducing postoperative pain, antiinflammation, antianaphylaxis, immunosuppression and so on[12, 24, 25]. The administration of dexamethasone during treatment of nonhematologic malignancy is at issue. The effect of dexamethasone on oncological outcomes has been investigated by another researchers. Obradovic MMS et al. reported that glucocorticoids increased the risk of breast cancer metastasis by activating glucocorticoid receptor[26]. Similarly, immunosuppressive dose of dexamethasone might enhance prostate cancer progression[27]. In rectal cancer patients with curative resection, there was an association between low-dose perioperative dexamethasone and poorer survival[28]. On the contrary, glucocorticoids perform a beneficial effect in certain solid tumors. Perioperatively administration of dexamethasone may improve survival in pancreatic adenocarcinoma patients[29]. A retrospective study noted that perioperatively dexamethasone had no effect on ovarian cancer recurrence[30]. The aforementioned studies implied that dosage, timing and frequency of dexamethasone played a decisive role in different oncological outcomes. Histopathological type of tumor and primary site of tumor were equally important. As a consequence, further researches are wanted to verify the effect of dexamethasone on other cancer.

A few studies suggested the correlation between administration of dexamethasone and survival in nonsmall lung cancer patients with lung cancer resection. One study noted that NSCLC patients treated with lung cancer resection may get survival benefit from perioperatively administration of dexamethasone[15]. A systematic analysis reported that glucocorticoids might have a deleterious effect in NSCLC patients. Another study indicated that dexamethasone was not associated with recurrence-free survival and overall survival after lung cancer resection for NSCLC patients[16]. These results are inconsistent and inadequate, and more studies are necessary. Our study provides more evidence to verify the association between intraoperatively administration of dexamethasone and survival in NSCLC patients as well as in some certain subgroup populations.

Lack of correlation between intraoperatively administration of dexamethasone and survival may be associated with the following several factors. On one hand, two factors may facilitate favorable effect of dexamethasone on survival for NSCLC patients. Dexamethasone reduces stress response which has a detrimental influence on host immune function[31]. In addition, dexamethasone can suppress proliferation of non-small cell lung cancer by inactivating estrogen[32]. On the other hand, intraoperatively administration of dexamethasone augments the risks of cancer recurrence and metastasis through several possible ways. Dexamethasone depresses host immune defense system by inhibiting the function of natural killer cell (NK), which takes part in tumor cytotoxicity[33]. Besides, low concentrations of dexamethasone not only induce proliferation of normal cells, but also induces proliferation of cancer cell in vitro, including glioma and astrocytoma[34]. Meanwhile, dexamethasone, as a long half-life glucocorticoid, may remain stable for 36-72 hours in humans[35]. Thus, the detrimental effects of dexamethasone might persist for a relatively long period of time in non-small cell lung cancer patients. As a consequence, these deleterious effects may be just right to counteract the favorable effects of dexamethasone to generate an overall neutral effect. 
This study has some limitations. Firstly, sample size was relatively small. Our study consisted of 148 patients receiving intraoperative dexamethasone and 675 patients who were not given dexamethasone. After propensity score matching, there were only 206 patients in the non-DEX group and 103 patients in the DEX group. Secondly, we performed a propensity score matching analysis to decrease the potential confounding effect of each variable. But we failed to take into consideration those unknown confounding factors. Thirdly, the date of cancer recurrence could be inaccuracy and we may overestimate the date of cancer recurrence. Because relapse may occur before relapse was detected by imaging or histopathological examination. Moreover, our study couldn't collect the details of intraoperative and postoperative opioids administration, so we couldn't assess the effect of opioids on survival. Finally, we couldn't avoid selection bias on account of retrospective analysis. This could be an important reason that we found no association between intraoperative dexamethasone and survival.

Our results show that there was no correlation between intraoperatively administration of dexamethasone and survival in NSCLC patients after lung cancer resection. However, we still don't know whether the correlation between intraoperatively dexamethasone and survival is coincidental. The exact effects of intraoperative dexamethasone on non-small cell lung cancer patients with lung cancer resection should be verified in further researches. An adequately powered randomized control trial (RCT) that has strict inclusion criteria and exclusion criteria will verify the effect of intraoperative dexamethasone on diseasefree survival and overall survival for non-small cell lung cancer patients.

\section{Conclusion}

There was no correlation between intraoperatively administration of dexamethasone and survival in nonsmall cell lung cancer (NSCLC) patients. Comparing with patients not receiving dexamethasone intraoperatively, patients receiving dexamethasone had improved disease-free survival in the subgroup of anesthetic time less than 2 hours. Intraoperatively administration of dexamethasone may improve overall survival in the subgroup of VATS.

Our results indicate that intraoperatively administration of dexamethasone has no impact on survival in NSCLC patients with lung cancer resection. In some certain patient populations, intraoperative administration of systemic dexamethasone is probably favorable. However, the effects and mechanisms of dexamethasone on non-small cell lung cancer should be verified in further research.

\section{Abbreviations}

ASA: American Society of Anesthesiologists; BMl: Body mass index; Cl: Confidence interval; COPD: Chronic obstructive pulmonary disease; DEX: Dexamethasone; DFS: Disease-free survival; GC: Glucocorticoids; HR: Hazards ratio; IV: Intravenous; NSCLC: Non-small cell lung cancer; OS: Overall survival; PCIA: Patient controlled intravenous analgesia; PCEA: Patient controlled epidural analgesia; PONV: Postoperative nausea and vomiting; RCT: Randomized control trial; SD: Standard Deviation; VATS: Video-assisted thoracic surgery. 


\section{Declarations}

Acknowledgements

Not applicable.

\section{Authors' contributions}

All authors had full access to all the data and take responsibility for the integrity of the data and accuracy of the data analysis. DTC, WAZ and FY helped design the study. HQZ, WX and QL helped conduct of the study and data collection. FY, GC and JDX helped conduct of the study, data analysis, interpretation of the results and manuscript preparation as co-authors. All authors have read and approved the final manuscript.

\section{Funding}

This work was supported by grants from National Natural Science Foundation of China (grant 81971057 to W.A.Z., and grant 81902490 to D.T.C.) and Natural Science Foundation of Guangdong province (grant 2018A030313092 to J.D.X).

\section{Availability of data and materials}

The datasets used or presented during this study are available from the corresponding author upon reasonable request.

\section{Ethics approval and consent to participate}

The medical study was approved by the Clinical Research Ethics Committee of Sun Yat-sen University Cancer Center. The informed consent was waived because of the retrospective nature of this study and no risk for the patients in this study.

\section{Consent for publication}

Not applicable.

\section{Competing interests}

The authors declare that they have no competing interests.

\section{References}

1. Hanahan D, Weinberg RA: Hallmarks of cancer: the next generation. Cell 2011, 144(5):646-674.

2. Moghissi K, Dixon K: Image-guided surgery and therapy for lung cancer: a critical review. Future oncology (London, England) 2017, 13(26):2383-2394. 
3. Nagasaka M, Gadgeel SM: Role of chemotherapy and targeted therapy in early-stage non-small cell lung cancer. Expert review of anticancer therapy 2018, 18(1):63-70.

4. Jewett A, Kos J, Kaur K, Safaei T, Sutanto C, Chen W, Wong P, Namagerdi AK, Fang C, Fong Y et al: Natural Killer Cells: Diverse Functions in Tumor Immunity and Defects in Preneoplastic and Neoplastic Stages of Tumorigenesis. Molecular therapy oncolytics 2020, 16:41-52.

5. Tamminga M, de Wit S, Van De Wauwer C, van den Bos H, Swennenhuis JF, Klinkenberg TJ, Hiltermann TJN, Andree KC, Spierings DCJ, Lansdorp PM et al: Release of circulating tumor cells during surgery for non-small cell lung cancer: are they what they appear to be? Clinical cancer research : an official journal of the American Association for Cancer Research 2019.

6. Milette S, Fiset PO, Walsh LA, Spicer JD, Quail DF: The innate immune architecture of lung tumors and its implication in disease progression. The Journal of pathology 2019, 247(5):589-605.

7. Shakhar G, Abudarham N, Melamed R, Schwartz Y, Rosenne E, Ben-Eliyahu S: Amelioration of operation-induced suppression of marginating pulmonary NK activity using poly IC: a potential approach to reduce postoperative metastasis. Annals of surgical oncology 2007, 14(2):841-852.

8. Latif MJ, Tan KS, Molena D, Huang J, Bott MJ, Park BJ, Adusumilli PS, Rusch VW, Bains MS, Downey RJ et al: Perioperative blood transfusion has a dose-dependent relationship with disease recurrence and survival in patients with non-small cell lung cancer. The Journal of thoracic and cardiovascular surgery 2019, 157(6):2469-2477.e2410.

9. Cata JP, Gottumukkala V, Thakar D, Keerty D, Gebhardt R, Liu DD: Effects of postoperative epidural analgesia on recurrence-free and overall survival in patients with nonsmall cell lung cancer. Journal of clinical anesthesia 2014, 26(1):3-17.

10. Hasegawa T, Oguri T, Osawa T, Sawa T, Osaga S, Okuyama T, Uchida M, Maeno K, Fukuda S, Nishie H et al: Opioid Dose and Survival of Patients with Incurable Nonsmall Cell Lung Cancer: A Prospective Cohort Study. Journal of palliative medicine 2018, 21(10):1436-1441.

11. Gan TJ, Diemunsch P, Habib AS, Kovac A, Kranke P, Meyer TA, Watcha M, Chung F, Angus S, Apfel CC et al: Consensus guidelines for the management of postoperative nausea and vomiting. Anesthesia and analgesia 2014, 118(1):85-113.

12. Son J, Yoon H: Factors Affecting Postoperative Nausea and Vomiting in Surgical Patients. Journal of perianesthesia nursing : official journal of the American Society of PeriAnesthesia Nurses 2018, 33(4):461-470.

13. Ai F, Zhao G, Lv W, Liu B, Lin J: Dexamethasone induces aberrant macrophage immune function and apoptosis. Oncology reports 2020, 43(2):427-436.

14. Chen $L$, Jondal M, Yakimchuk K: Regulatory effects of dexamethasone on NK and T cell immunity. Inflammopharmacology 2018, 26(5):1331-1338.

15. Huang WW, Zhu WZ, Mu DL, Ji XQ, Nie XL, Li XY, Wang DX, Ma D: Perioperative Management May Improve Long-term Survival in Patients After Lung Cancer Surgery: A Retrospective Cohort Study. Anesthesia and analgesia 2018, 126(5):1666-1674. 
16. Cata JP, Jones J, Sepesi B, Mehran RJ, Rodriguez-Restrepo A, Lasala J, Feng L, Gottumukkala V: Lack of Association Between Dexamethasone and Long-Term Survival After Non-Small Cell Lung Cancer Surgery. Journal of cardiothoracic and vascular anesthesia 2016, 30(4):930-935.

17. Wagner BJ, Buettner R: [Immunohistochemical and molecular pathological diagnosis of lung carcinoma]. Der Pathologe 2020.

18. Kandathil A, Kay FU, Butt YM, Wachsmann JW, Subramaniam RM: Role of FDG PET/CT in the Eighth Edition of TNM Staging of Non-Small Cell Lung Cancer. Radiographics : a review publication of the Radiological Society of North America, Inc 2018, 38(7):2134-2149.

19. Rubin DB, Thomas N: Matching using estimated propensity scores: relating theory to practice. Biometrics 1996, 52(1):249-264.

20. Schulte PJ, Mascha EJ: Propensity Score Methods: Theory and Practice for Anesthesia Research. Anesthesia and analgesia 2018, 127(4):1074-1084.

21. Kim J, Kaufman JS, Bang H: Graphing Ratio Measures on Forest Plot. Journal of the American College of Cardiology 2018, 71(5):585-586.

22. Cari L, De Rosa F, Nocentini G, Riccardi C: Context-Dependent Effect of Glucocorticoids on the Proliferation, Differentiation, and Apoptosis of Regulatory T Cells: A Review of the Empirical Evidence and Clinical Applications. International journal of molecular sciences 2019, 20(5).

23. Bertoli S, Picard M, Berard E, Griessinger E, Larrue C, Mouchel PL, Vergez F, Tavitian S, Yon E, Ruiz J et al: Dexamethasone in hyperleukocytic acute myeloid leukemia. Haematologica 2018, 103(6):988998.

24. De Oliveira GS, Jr., Almeida MD, Benzon HT, McCarthy RJ: Perioperative single dose systemic dexamethasone for postoperative pain: a meta-analysis of randomized controlled trials. Anesthesiology 2011, 115(3):575-588.

25. Adcock IM, Mumby S: Glucocorticoids. Handbook of experimental pharmacology 2017, 237:171-196.

26. Obradovic MMS, Hamelin B, Manevski N, Couto JP, Sethi A, Coissieux MM, Munst S, Okamoto R, Kohler H, Schmidt A et al: Glucocorticoids promote breast cancer metastasis. Nature 2019, 567(7749):540-544.

27. Jain S, Dash P, Minz AP, Satpathi S, Samal AG, Behera PK, Satpathi PS, Senapati S:

Lipopolysaccharide (LPS) enhances prostate cancer metastasis potentially through NF-kappaB activation and recurrent dexamethasone administration fails to suppress it in vivo. The Prostate 2019, 79(2):168-182.

28. Yu HC, Luo YX, Peng H, Kang L, Huang MJ, Wang JP: Avoiding perioperative dexamethasone may improve the outcome of patients with rectal cancer. European journal of surgical oncology : the journal of the European Society of Surgical Oncology and the British Association of Surgical Oncology 2015, 41(5):667-673.

29. Call TR, Pace NL, Thorup DB, Maxfield D, Chortkoff B, Christensen J, Mulvihill SJ: Factors associated with improved survival after resection of pancreatic adenocarcinoma: a multivariable model. Anesthesiology 2015, 122(2):317-324. 
30. De Oliveira GS, Jr., McCarthy R, Turan A, Schink JC, Fitzgerald PC, Sessler DI: Is dexamethasone associated with recurrence of ovarian cancer? Anesthesia and analgesia 2014, 118(6):1213-1218.

31. Shirazi SN, Friedman AR, Kaufer D, Sakhai SA: Glucocorticoids and the Brain: Neural Mechanisms Regulating the Stress Response. Advances in experimental medicine and biology 2015, 872:235-252.

32. Wang LJ, Li J, Hao FR, Yuan Y, Li JY, Lu W, Zhou TY: Dexamethasone suppresses the growth of human non-small cell lung cancer via inducing estrogen sulfotransferase and inactivating estrogen. Acta pharmacologica Sinica 2016, 37(6):845-856.

33. Misale MS, Witek Janusek L, Tell D, Mathews HL: Chromatin organization as an indicator of glucocorticoid induced natural killer cell dysfunction. Brain, behavior, and immunity 2018, 67:279289.

34. Cho KA, Kim HJ, Kim YH, Park M, Woo SY: Dexamethasone Promotes Keratinocyte Proliferation by Triggering Keratinocyte Growth Factor in Mast Cells. International archives of allergy and immunology 2019, 179(1):53-61.

35. Czock D, Keller F, Rasche FM, Haussler U: Pharmacokinetics and pharmacodynamics of systemically administered glucocorticoids. Clinical pharmacokinetics 2005, 44(1):61-98.

\section{Figures}




\section{Figure 1}

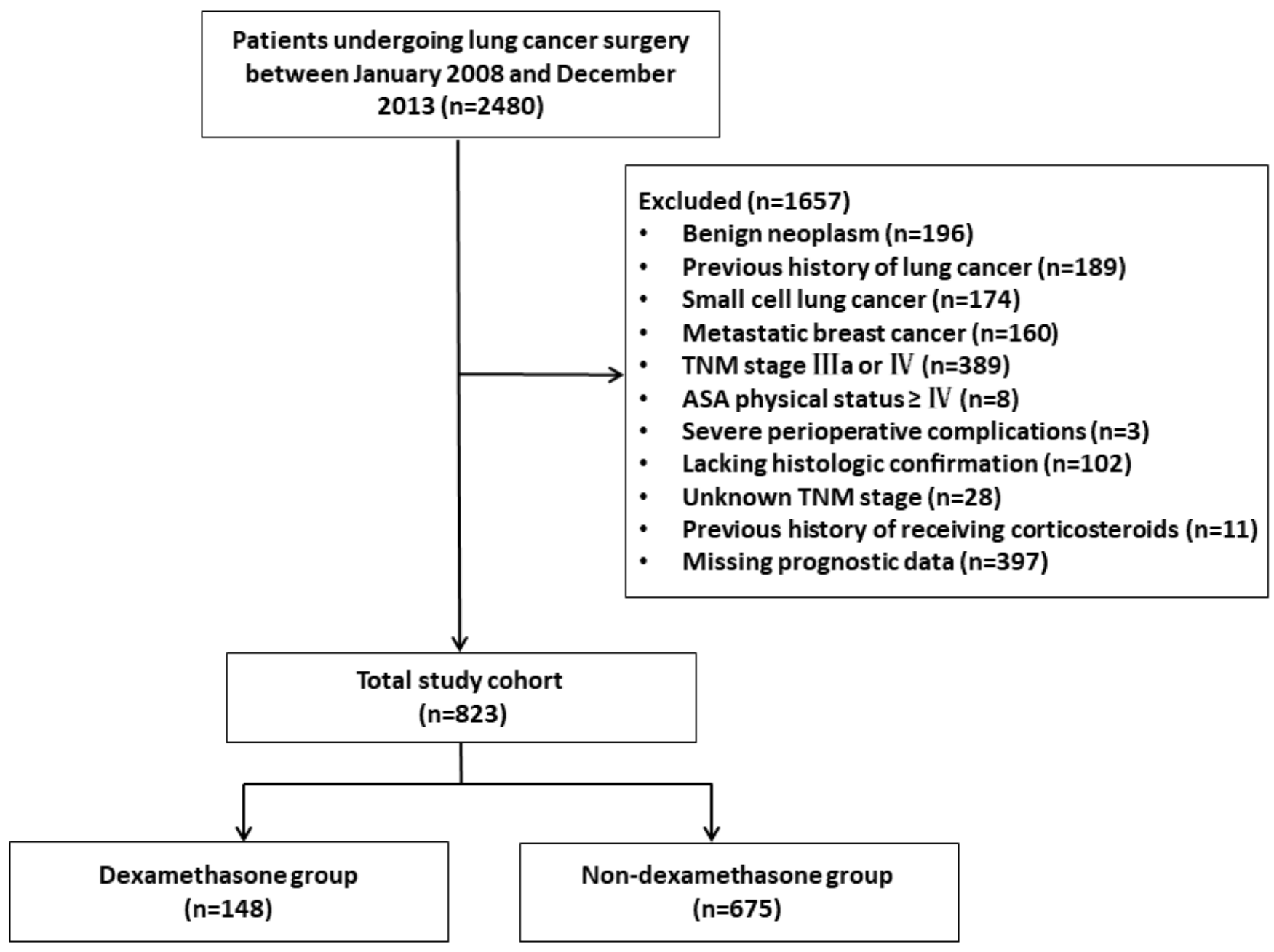

Figure 1

Flowchart of study selection. 
Figure 2
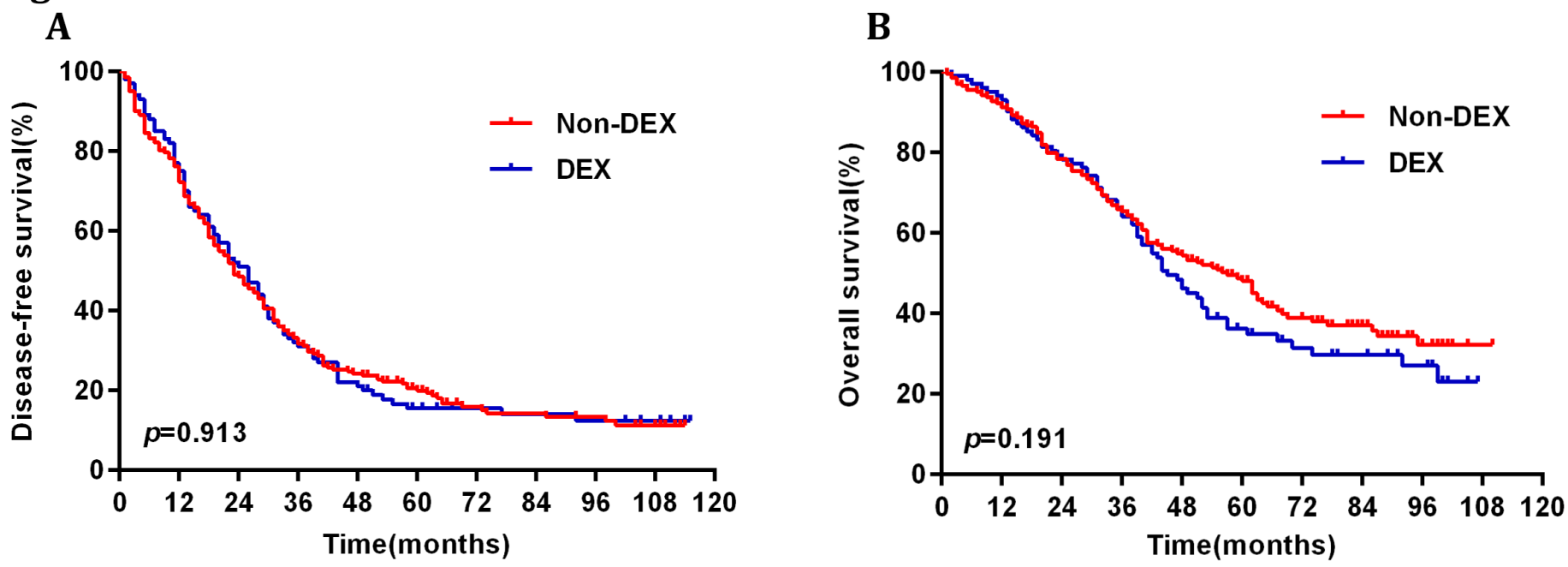

Figure 2

The Kaplan-Meier curves of the intraoperative dexamethasone (DEX) and non-DEX groups in the propensity matched cohort. (A) Disease-free survival. (B) Overall survival. DEX, dexamethasone.

Figure 3

A

Disease-free survival

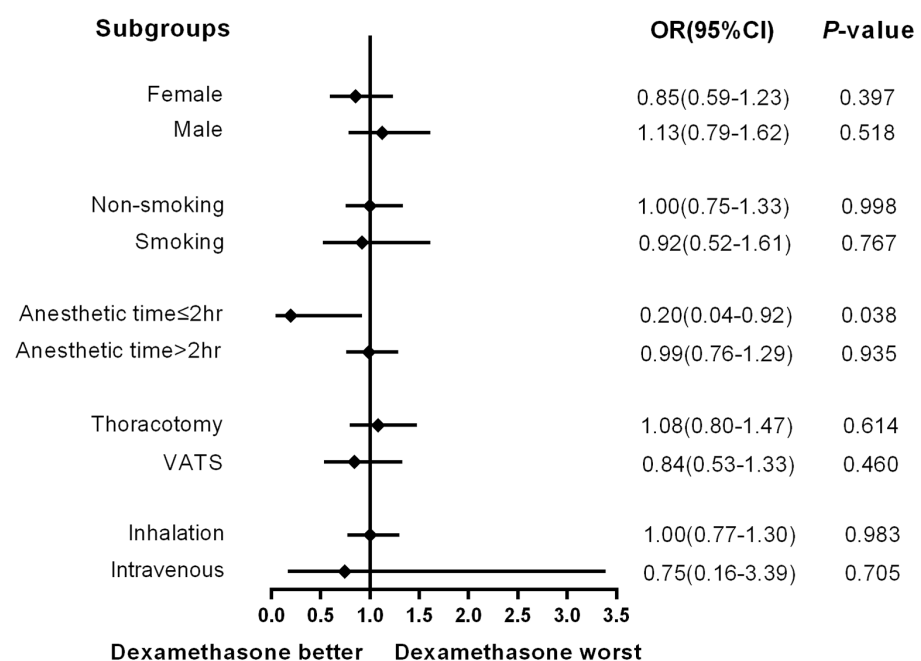

B

Overall survival

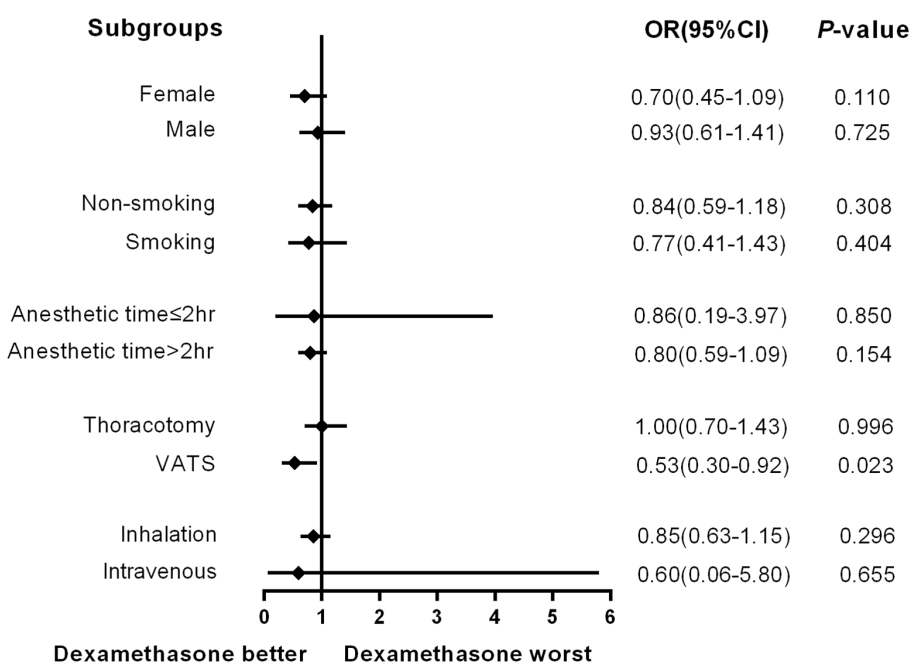

\section{Figure 3}

Subgroup analysis of disease-free survival (A) and overall survival (B) according to the risk factors of postoperative nausea and vomiting. $\mathrm{Cl}$, confidence interval; OR, odd ratio; VATS, video-assisted thoracic surgery. 